\title{
Automated Electrospray Ionization FT-ICR Mass Spectrometry for Petroleum Analysis
}

\author{
Sunghwan Kim, ${ }^{a}$ Ryan P. Rodgers, ${ }^{\mathrm{b}}$ Greg T. Blakney, \\ Christopher L. Hendrickson, ${ }^{\text {and Alan G. Marshall }}{ }^{\mathrm{b}}$ \\ ${ }^{a}$ Korean Basic Science Institute, Chungcheongbuk-Do, Republic of Korea \\ ${ }^{\mathrm{b}}$ Ion Cyclotron Resonance Program, National High Magnetic Field Laboratory, Florida State University, \\ Tallahassee, Florida
}

Analysis of petroleum samples at the molecular level by Fourier transform ion cyclotron resonance mass spectrometry (FT-ICR MS) typically requires a prolonged accumulation of ions and/or summing up a large number of scans. Here, a chip-based micro-ESI system (Advion NanoMate, Ithaca, NY) has been successfully automated in combination with FT-ICR MS analysis of petroleum samples. A foil-sealed 96-well glass plate prevents solvent evaporation, with no visible loss of sample after $20 \mathrm{~h}$ of continuous operation. Mass spectra obtained from the same sample but taken from different wells after various time delays were very similar. Data from replicate samples in different wells could be combined to enhance mass spectral signal-to-noise ratio and dynamic range. Furthermore, the automated data acquisition eliminates sample carryover, and produces heteroatom class distribution, double-bond equivalents (DBE), and carbon number very similar to those from the conventional (manual) micro-ESI experiments. (J Am Soc Mass Spectrom 2009, 20, 263-268) (C) 2009 Published by Elsevier Inc. on behalf of American Society for Mass Spectrometry

$\mathrm{F}$ ourier Transform ion cyclotron resonance mass spectrometry (FT-ICR MS) offers both high-resolution (mass resolving power $=\mathrm{m} / \Delta \mathrm{m}_{50 \%}>400,000$, in which $\Delta \mathrm{m}_{50 \%}$ is magnitude-mode FT-ICR mass spectral peak full width at half-maximum peak height) and high mass accuracy ( $<400 \mathrm{ppb}$ rms error). FT-ICR MS resolving power of 3300,000 for a $\sim 1 \mathrm{kDa}$ peptide has been reported [1]. Elemental composition may be assigned uniquely from sufficiently accurate mass measurement for a well-resolved peak [2]. The identified compositions may be further sorted by various tools including hydrogen deficiency $(\mathrm{z})$ based on $\mathrm{C}_{\mathrm{c}} \mathrm{H}_{2 \mathrm{c}+\mathrm{z}} \mathrm{N}_{\mathrm{n}} \mathrm{O}_{\mathrm{o}} \mathrm{S}_{\mathrm{s}}$ or double-bond equivalents (DBE = number of rings plus double bonds to carbon), Kendrick mass defect versus Kendrick mass [3, 4], or van Krevelen analysis (e.g., $\mathrm{H} / \mathrm{C}$ ratio versus $\mathrm{O} / \mathrm{C}$ or $\mathrm{N} / \mathrm{C}$ or $\mathrm{S} / \mathrm{C}$ ratio) [5]. Therefore, it is not surprising to find that FT-ICR MS has been successfully applied to study complex natural organic mixtures, including metabolites [6], vegetable oils [7], wine [8], explosives [9], coal extracts [10], and humic materials [11, 12].

An important application of FT-ICR MS is the emerging field of "petroleomics" [13], namely, the idea that the detailed chemical composition should correlate with (and ultimately predict) the properties and behavior of petroleum and its products. FT-ICR MS-based chemical composition has already enabled characterization of asphaltenes $[14,15]$, distillates $[16,17]$, and mined/steam assisted

Address reprint requests to Dr. A. G. Marshall, Ion Cyclotron Resonance Program, National High Magnetic Field Laboratory, Florida State University, 1800 E. Paul Dirac Drive, Tallahassee, FL 32310-4005, USA. E-mail: marshall@magnet.fsu.edu gravity drainage [18] at the molecular level. However, a single petroleum FT-ICR mass spectrum can contain up to $\sim 50,000$ peaks [19], whereas detection is typically limited to $\sim 1,000,000$ ions at a time. Thus, the number of ions corresponding to each resolved mass-to-charge ratio is relatively small so that it is often desirable to sum at least 100 time-domain transients to increase signal-to-noise ratio and dynamic range. Data acquisition for a single sample can thus take up to an hour.

Fortunately, most petroleum samples present similar $m / z$ range, so that the same experimental conditions apply to a series of related samples, i.e., such analyses are good candidates for automation, for improved reliability and throughput (e.g., overnight). Chip-based automated electrospray MS has been successfully applied to study carbohydrates [20], metabolites [21], peptides/proteins [22], and lipids [23].

Recently, chip-based electrospray ionization (ESI) has been used to measure total acid number (TAN) of petroleum samples by low-resolution mass analysis [24], with improved throughput and reduced sample consumption. Carryover from one sample to the next is eliminated because the automated system supplies a new spray tip and nozzle for each sample. Here, we report a fully automated chip-based micro-electrospray ultrahigh-resolution FT-ICR MS system for automated/ unattended/remote FT-ICR MS data collection for complex petroleum samples. Performance of the system has been tested for reproducibility over extended ( $>20 \mathrm{~h}$ ) data collection, and means for detecting and halting
(C) 2009 Published by Elsevier Inc. on behalf of American Society for Mass Spectrometry. $1044-0305 / 09 / \$ 32.00$

doi:10.1016/j.jasms.2008.10.001
Published online October 9, 2008 Received September 2, 2008 Revised September 30, 2008 Accepted October 1, 2008 
faulty data acquisitions are described. The system performs as well as fully attended manual data collection.

\section{Experimental}

Ten petroleum samples were chosen to test the ability of the automation procedure to provide high quality mass spectra from a wide range of samples: bitumens, heavy vacuum gas oils, and light $\left(343-375^{\circ} \mathrm{C}\right.$ ) and heavy distillates $\left(500-525^{\circ} \mathrm{C}\right)$. All samples were dissolved to 1 $\mathrm{mg} / \mathrm{mL}$ in toluene:methanol (50:50 vol/vol) and spiked with $1 \%$ (by volume) acetic acid before sample loading. Glass plates with 96 wells were purchased from Zinsser Analytic (Frankfurt, German). The petroleum samples were each placed in three or five glass wells (see below) ( $\sim 200 \mu \mathrm{L}$ per well-more could be used if required) and sealed with aluminum foil by an in-house designed clamp (see the Results and Discussion section). The samples were introduced sequentially by a NanoMate 100 (Advion Biosciences, Inc., Ithaca, NY). The NanoMate was operated in positive-ion mode with 1500-2000 V spray voltage and 0.5 psi gas pressure. A custom-built FT-ICR mass spectrometer equipped with a $220 \mathrm{~mm}$ horizontal room-temperature bore $9.4 \mathrm{~T}$ magnet [25] was used in these experiments. Ions generated in the ESI source region are first accumulated in an external linear octopole ion trap, typically for 1 to $5 \mathrm{~s}$, and transferred through rf-only multipoles to a $10 \mathrm{~cm}$ diameter, $30 \mathrm{~cm}$ long open cylindrical Penning ion trap [26].

Multipoles [25] were typically operated at $1.7 \mathrm{MHz}$ at an rf amplitude of $170 \mathrm{~V}_{p-p}$. After ions were excited in the trap by broadband frequency-sweep (chirp) dipolar excitation $(70-641 \mathrm{kHz}$ at a sweep rate of $150 \mathrm{~Hz} / \mu \mathrm{s}$ and amplitude, $440 \mathrm{~V}_{p-p}$ ), direct mode image current detection was performed to yield $4 \mathrm{M}$ word timedomain data. Time domain datasets were co-added (100 acquisitions), Hanning apodized, and zero-filled once before fast Fourier transformation and magnitude calculation. Frequency was converted to $m / z$ ratio by the quadrupolar electric trapping potential approximation [27, 28].

The instrument was controlled by an in-house Predator data station [29]. The Predator system features 17 channels of analog voltage $( \pm 10 \mathrm{~V}$, sixteen 16-bit voltages and one 12-bit voltage), 18 TTL triggers, and a 6-channel high power voltage amplifier $( \pm 200 \mathrm{~V})$. Dipolar excitation circuitry is implemented with a commercial 16-bit arbitrary waveform generator that drives a commercial broadband amplifier. A commercial balun transformer generates discrete dipolar channels to the ICR cell. Ion detection circuitry includes a Pacific Northwest National Laboratory low noise differential preamplifier coupled to a commercial low pass filter and finally recorded with a 14-bit commercial digitizer. Control software for the above data station was developed in-house and is written in the $\mathrm{C}$ programming language.

\section{Results and Discussion}

\section{Synchronizing the NanoMate and the Predator Data Station}

Acquisition begins by separate manual (mouse click) start of the Predator and NanoMate methods. The Predator method is contained within a TCL script such that the initial data collection proceeds without the typical wait state and associated external triggering. After the mass spectrometer has collected the specified number of scans, Predator sends a signal to the NanoMate to stop the spray and move to the next sample. While the NanoMate loads the new sample, the Predator data station is set to single external trigger mode and progresses to a wait state. The NanoMate discards the last ESI tip, gets a new tip and sample, starts spraying into a new nozzle, waits for $2 \mathrm{~s}$, and sends a trigger back to the data station to initiate the next cycle. Signals to and from the NanoMate are contact closure, (triggered by completing the circuit or a TTL low signal).

Each sample list is built in the NanoMate software. A NanoMate method was written to import a trigger to end the spray and also to export a trigger $2 \mathrm{~s}$ after the beginning of each new spray. The capability to receive a trigger to end a spray and send a signal at the beginning of a new spray is available from the

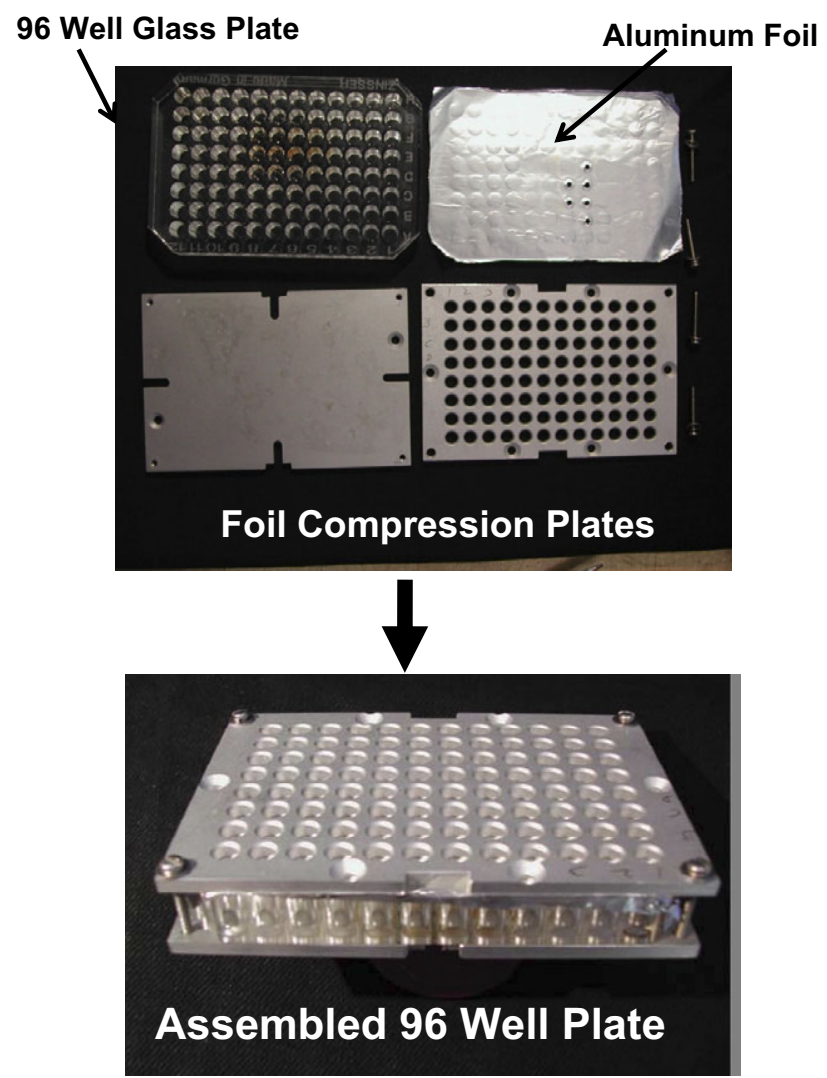

Figure 1. Photos of the unassembled (top) and assembled (bottom right) multiple-sample holder, and the holder installed in the NanoMate robot (bottom left). 


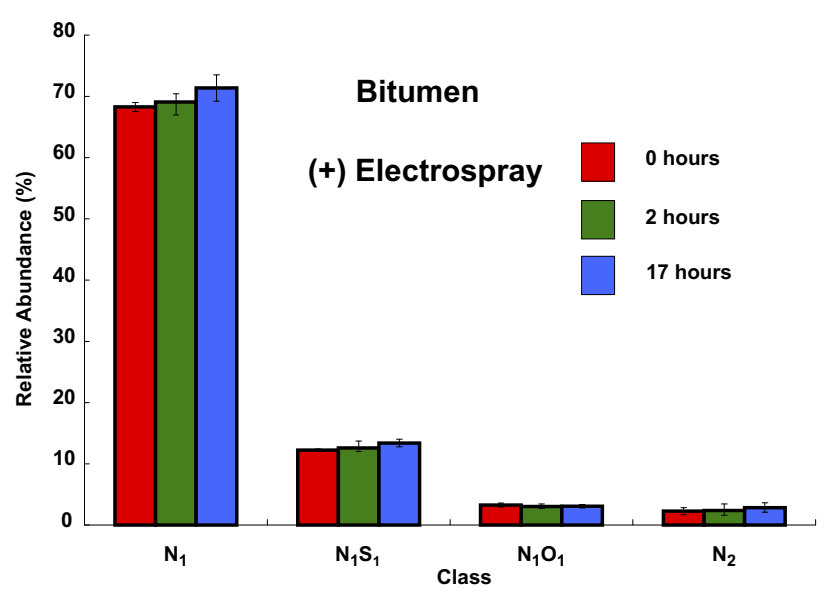

Figure 2. Relative abundances for each of the four most abundant heteroatom classes for bitumen replicate samples analyzed by positive-ion ESI 9.4 T FT-ICR MS after 0, 2, and $17 \mathrm{~h}$. Note the excellent reproducibility over time.

vendor program, but must be enabled within the user method.

\section{6-Well Plate with Reduced Sample Evaporation}

In petroleomic analyses, samples are typically prepared in volatile organic solvents such as toluene and methanol. Therefore, evaporative loss during extended automated data collection must be prevented. Moreover, such solvents are incompatible with the materials used in typical sealant systems designed for biological analytes in 96-well plates.
The Advion NanoMate system can draw $\sim 15 \mu \mathrm{L}$ of sample into its polymer-based spray tip. Consequently, for longer ESI spray periods, multiple sealed sample compartments must be available because once pierced, the original compartment will suffer from unwanted evaporative loss. We therefore designed compression plates (Figure 1) to prevent solvent evaporation. Aluminum top and bottom plates each have 96 holes to mate with a 96-well glass plate sandwiched between them. Four bolts between the aluminum plates compress a sheet of aluminum foil placed between the top aluminum plate and the glass plate. Aluminum foil was chosen because it is inert to organic solvents; a NanoMate collection tip can easily puncture a hole to take up sample liquid without damage to the tip; and compression of the foil serves to seal each compartment. The assembled 96-well plate (see Figure 1) is then installed in the NanoMate robot.

An Athabasca bitumen petroleum sample was loaded into 9 of the 96 wells and sealed. To evaluate sample evaporation, samples were analyzed in triplicate after 0,2 , and $17 \mathrm{~h}$. The automated data collection yielded ESI FT-ICR mass spectra with average mass resolving power, $\mathrm{m} / \Delta \mathrm{m}_{50 \%} \approx 500,000$, at $\mathrm{m} / \mathrm{z}=400$, resulting in $\sim 300 \mathrm{ppb}$ rms mass accuracy after internal calibration. The relative abundances (with error bars for triplicate runs) for the four most abundant heteroatom classes (e.g., $\mathrm{N}_{1}$ denotes the summed relative abundances of all species containing one nitrogen atom) are shown in Figure 2. The sealed glass plate showed no sign of sample evaporation after $17 \mathrm{~h}$ of operation, and the variation of relative abundances with time was

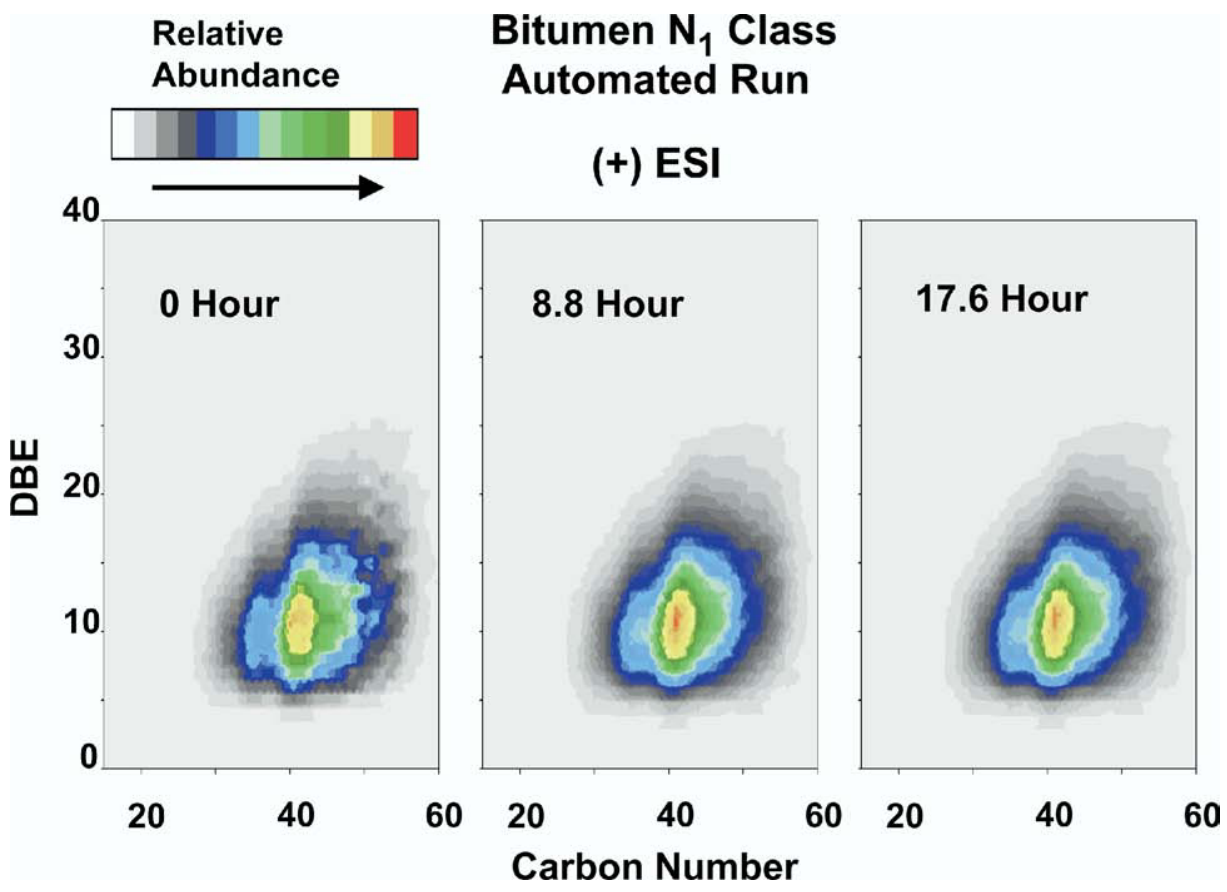

Figure 3. Isoabundance-contoured plots of double-bond equivalents (DBE) versus carbon number for members of the $\mathrm{N}_{1}$ heteroatom class from Athabasca bitumen. Note the high reproducibility over $17 \mathrm{~h}$. 
within the range of replicate errors from a given time delay.

\section{System Reliability}

To test the robustness of the glass plate design and the NanoMate robot for petroleum analysis, 10 petroleum samples were run from five different wells each (100 time-domain transients summed per run for a total of 5000 scans) over a $22 \mathrm{~h}$ period. Figure 3 shows isoabundancecontoured images of double-bond equivalents (DBE) versus carbon number for the $\mathrm{N}_{1}$ (i.e., the most abundant) class species from Athabasca bitumen, from positive-ion ESI 9.4 T FT-ICR mass spectra starting at 0, 8.8 and $17.6 \mathrm{~h}$. The other nine petroleum samples gave similarly reproducible results (not shown). We conclude that the automated system presented here is suitable for multiple/ unattended analyses.

\section{Enhanced Dynamic Range by Automation}

Figure 4 contains mass scale-expanded segments $(701.3<$ $\mathrm{m} / \mathrm{z}<701.7$ ) of ESI 9.4 T FT-ICR mass spectra of a petroleum light distillate sample taken during the $22 \mathrm{~h}$ unattended series of experiments discussed above. In anticipation of the occurrence of failed runs (e.g., clogged tip, bad seal on the glass plate, inconsistent electrospray) each sample was run five times (from separate wells) $\sim 4 \mathrm{~h}$ apart. Figure 4 (top) is from the first of the five runs of the light distillate, for which most of the ion masses are below $700 \mathrm{Da}$. One of the five replicate runs failed, most likely due to a clog in the ESI nozzle. However, the benefit of unattended/automated analysis is that multiple runs of the same sample can be easily accommodated. In this example, four of the five runs could be co-added for a factor of 2 increase in

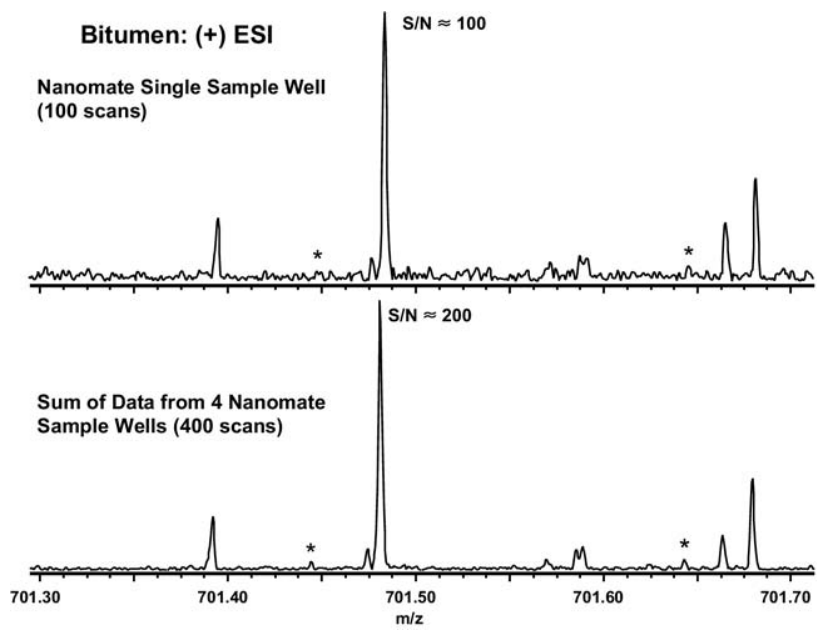

Figure 4. Mass scale-expanded segments $(701.3<m / z<701.7)$ of a positive-ion ESI $9.4 \mathrm{~T}$ FT-ICR mass spectrum of Athabasca bitumen. Top: 100 scans from a single sample well. Bottom: 400 scans summed from the data from four sample wells. The 2-fold improvement in $\mathrm{S} / \mathrm{N}$ ratio makes it possible to visually distinguish weak signals (asterisk) from noise.

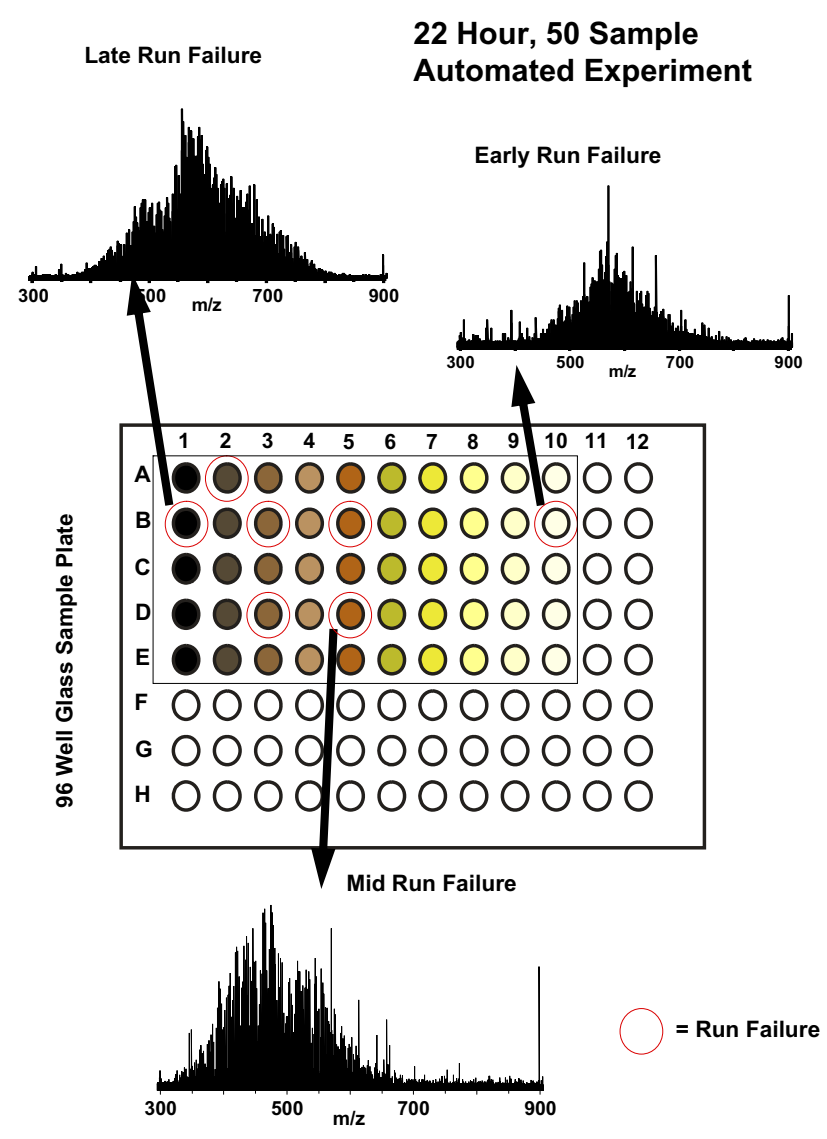

Figure 5. Failure analysis from a 22-h automated data acquisition from 50 wells containing five replicates of each of 10 different petroleum samples (see text).

signal-to-noise ratio with no associated loss of resolution or mass accuracy (Figure 4, bottom). The increased signal-to-noise ratio enhances the dynamic range of the analysis. For example, the weak signals (denoted by an asterisk) cannot be distinguished from noise after 100 time-domain transients (Figure 4, top), but become visually distinguishable after 400 transients (Figure 4, bottom).

\section{Run Failures During Automated/Unattended Run}

Of the 50 samples (10 petroleum samples $\times 5$ runs, each 100 time-domain transients) analyzed over $22 \mathrm{~h}$, seven were unsuccessful. Based on the $86 \%$ success rate of the automated analysis, triplicate analyses should suffice to ensure at least one high-quality ESI FT-ICR mass spectrum per petroleum source. Analysis of the seven failed petroleum samples showed no clear signs of sample or time-dependent failure (Figure 5). However, analysis of the broadband mass spectra collected from each well revealed that of the expected early, middle, and late run failures, four failed within the first few (of 100) timedomain data acquisitions, two failed in the middle of the run, and one failed late in the run. Thus, it is possible to implement data-dependent co-add software to halt data acquisition for a given well if consecutive 
scans fail to reach a predefined signal-to-noise ratio threshold, to eliminate time lost to runs that fail soon after data acquisition begins.

\section{Comparison Between Automated and Conventional Micro ESI Analyses}

The NanoMate and our in-house micro-ESI sources have different spray tip configuration, e.g., lower voltage applied to the chip-based automated ESI system. Nevertheless, the results from both systems are consistent within experimental error. For example, Figure 6 shows isoabundance-contoured plots of double-bond equivalents (DBE) versus carbon number for the (highly abundant) $\mathrm{N}_{1}$ class of a bitumen (top), (less abundant) light distillate $\mathrm{N}_{1}$ (middle), and heavy distillate $\mathrm{N}_{1} \mathrm{~S}_{2}$ (bottom) classes. The spray tip and capillary of our conventional microESI source require frequent cleaning to avoid potential carryover between successive samples. However, carryover is inherently eliminated with
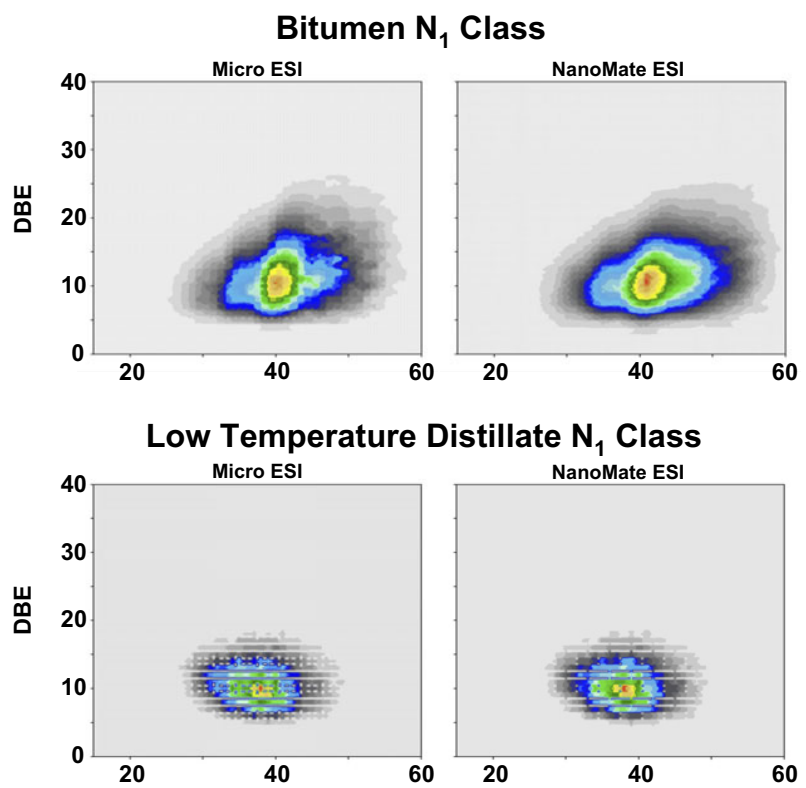

High Temperature Distillate $\mathrm{N}_{1} \mathrm{~S}_{2}$ Class

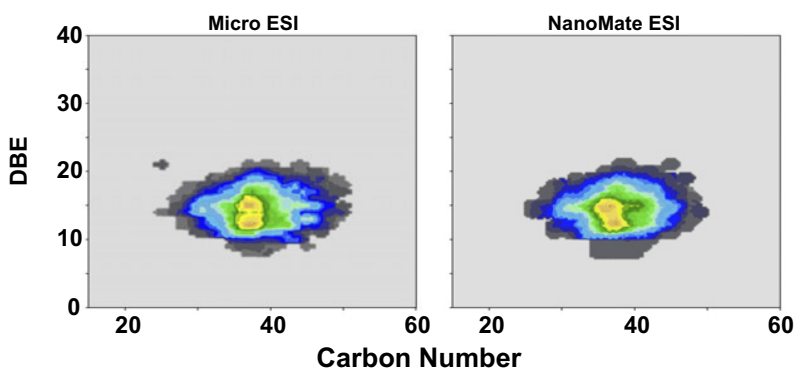

Figure 6. Isoabundance-contoured plots of double-bond equivalents (DBE) versus carbon number for three different petroleum samples and heteroatom classes (top, middle, bottom), based on positive-ion 9.4 T FT-ICR mass spectra acquired by manual micro-ESI (left) and NanoMate automated ESI (right). The agreement between data from the two ESI sources is excellent. the NanoMate because a new spray tip and nozzle are supplied for each sample well. Sample throughput is increased by both automation and eliminating the need for repeated cleaning of the ESI source.

\section{Conclusions}

A NanoMate system with a foil-sealed 96-well glass plate has been implemented and tested for automated ESI FT-ICR mass analysis of petroleum samples. The sealed 96 wells showed no evaporative loss of sample after $20 \mathrm{~h}$. The automated system exhibited excellent reproducibility (heteroatom class distribution, DBE, and carbon number) during a $22 \mathrm{~h}$ unattended/automated experiment, during which 10 different petroleum samples were run five times each (from separate wells) at $\sim 4 \mathrm{~h}$ time intervals for a total of 5000 individual time-domain transient data acquisitions. The success rate for the automated experiment was $\sim 86 \%$; ergo, triplicate analysis should suffice to ensure acquisition of high-quality mass spectra for each sample. Signal-to-noise ratio and dynamic range may be enhanced by co-adding data from (successful) acquisitions from multiple wells for the sample. Although the present experiments were performed with an in-house data station, the automation can be accomplished with other commercial data stations that send and receive external triggers. In the future, we shall expand the automation to negative-ion ESI and implement data-dependent co-addition to abort runs that fail before completion. Finally, the present configuration can obviously be coupled with FT-ICR MS analyses of electrosprayed samples of other analytes in other solvents.

\section{Acknowledgments}

The authors thank Daniel McIntosh and John P. Quinn for designing and machining the custom parts required for automated analysis. They thank Dr. Logan Mackay for helpful discussions. They acknowledge support for this work by NSF Division of Materials Research through DMR-0654118 and the State of Florida. SK acknowledges support by KBSI grant (N28085).

\section{References}

1. He, F.; Hendrickson, C. L.; Marshall, A. G. Baseline Mass Resolution of Peptide Isobars: A New Record for Molecular Mass Resolution. Anal. Chem. 2001, 73, 647-650.

2. Kim, S.; Rodgers, R. P.; Marshall A. G. Truly "Exact" Mass: Elemental Composition Can Be Determined Uniquely from Molecular Mass Measurement at Similar to $0.1 \mathrm{mDA}$ Accuracy for Molecules up to $500 \mathrm{Da}$. Int. J. Mass Spectrom. 2006, 251, 260-265.

3. Kendrick, E. A Mass Scale Based on $\mathrm{CH}_{2}=14.0000$ for High Resolution Mass Spectrometry of Organic Compounds. Anal. Chem. 1963, 35, 2146-2154.

4. Hughey, C. A.; Hendrickson, C. L.; Rodgers, R. P.; Marshall, A. G. Kendrick Mass Defect Spectrum: A Compact Visual Analysis for Ultra-High Resolution Broadband Mass Spectra. Anal. Chem. 2001, 73, 4676-4681.

5. Kim, S.; Kramer, R. W.; Hatcher, P. G. Graphical Method for Analysis of Ultrahigh-Resolution Broadband Mass Spectra of Natural Organic Matter, the van Krevelen Diagram. Anal. Chem. 2003, 75, 5336-5344.

6. Dettmer, K.; Aronov, P. A.; Hammock, B. D. Mass Spectrometry-Based Metabolomics. Mass Spectrom. Rev. 2007, 26, 51-78.

7. Wu, Z.; Rogers, R. P.; Marshall, A. G. Characterization of Vegetable Oils Detailed Compositional Fingerprints Derived from Electrospray Ioniza- 
tion Fourier Transform Ion Cyclotron Resonance Mass Spectrometry. J. Agric. Food Chem. 2004, 52, 5322-5328.

8. Cooper, H. J.; Marshall, A. G. Electrospray Ionization Fourier-Transform Ion Cyclotron Resonance Mass Spectrometric Analysis of Wine. J. Agric. Food Chem. 2001, 49, 5710-5718.

9. Wu, Z.; Hendrickson, C. L.; Rodgers, R. P.; Marshall, A. G. Compositional Analysis of Military Explosives by Electrospray Ionization Fourier Transform Negative Ion Cyclotron Resonance Mass Spectrometry. Anal. Chem. 2002, 74, 1879-1883.

10. Wu, Z.; Rodgers, R. P.; Marshall, A. G. Compositional Determination of Acidic Species in Illinois \#6 Coal Extracts by Electrospray Ionization Fourier Transform Ion Cyclotron Resonance Mass Spectrometry. Energy Fuels 2004, 18, 1424-1428.

11. Kim, S.; Simpson, A. J.; Kujawinski, E. B.; Freitas, M. A.; Hatcher, P. G. High Resolution Electrospray Ionization Mass Spectrometry and 2D Solution NMR for the Analysis of Dom Extracted by $\mathrm{C}_{18}$ Solid Phase Disk. Org. Geochem. 2003, 34, 1325-1335

12. Hockaday, W. C.; Grannas, A. M.; Kim, S.; Hatcher, P. G. Direct Molecular Evidence for the Degradation and Mobility of Black Carbon in Soils from Ultrahigh-Resolution Mass Spectral Analysis of Dissolved Organic Matter from a Fire-Impacted Forest Soil. Org. Geochem. 2006, 37, 501-510

13. Marshall, A. G.; Rodgers, R. P. Petroleomics: The Next Grand Challenge for Chemical Analysis. Acc. Chem. Res. 2004, 37, 53-59.

14. Klein, G. C.; Kim, S.; Rodgers, R. P.; Marshall, A. G. Mass Spectral Analysis of Asphaltenes. I. Compositional Differences between Pressure-Drop and Solvent-Drop Asphaltenes Determined by Electrospray Ionization Fourier Transform Ion Cyclotron Resonance Mass Spectrometry. Energy Fuels 2006, 20, 1965-1972

15. Klein, G. C.; Kim, S.; Rodgers, R. P.; Marshall, A. G.; Yen, A. Mass Spectral Analysis of Asphaltenes. II. Detailed Compositional Comparison of Asphaltenes Deposit to Its Crude Oil Counterpart for Two Geographically Different Crude Oils by ESI FT-ICR MS. Energy Fuels 2006, 20, 1973-1979.

16. Fu, J. M.; Kim, S.; Rodgers, R. P.; Hendrickson, C. L.; Marshall, A. G.; Qian, K. N. Nonpolar Compositional Analysis of Vacuum Gas Oil Distillation Fractions by Electron Ionization Fourier Transform Ion Cyclotron Resonance Mass Spectrometry. Energy Fuels 2006, 20, 661667.

17. Stanford, L. A.; Kim, S.; Rodgers, R. P.; Marshall, A. G. Characterization of Compositional Changes in Vacuum Gas Oil Distillation Cuts by Electrospray Ionization Fourier Transform-Ion Cyclotron Resonance (FT-ICR) Mass Spectrometry. Energy Fuels 2006, 20, 1664-1673.

18. Schaub, T. M.; Jennings, D. W.; Kim, S.; Rodgers, R. P.; Marshall, A. G. Heat-Exchanger Deposits in an Inverted Steam-Assisted Gravity Drainage Operation. Part 2. Organic Acid Analysis by Electrospray Ionization
Fourier Transform Ion Cyclotron Resonance Mass Spectrometry. Energy Fuels 2007, 21, 185-194.

19. Schaub, T. M.; Hendrickson, C. L.; Horning, S.; Quinn, J. P.; Senko, M. W.; Marshall, A. G. High-Performance Mass Spectrometry: Fourier Transform Ion Cyclotron Resonance at 14.5 Tesla. Anal. Chem. 2008, 80, 3985-3990.

20. Zamfir, A.; Vakhrushev, S.; Sterling, A.; Niebel, H. J.; Allen, M. Peter-Katalinic, J. Fully Automated Chip-Based Mass Spectrometry for Complex Carbohydrate System Analysis. Anal. Chem. 2004, 76, $2046-$ 2054.

21. Trunzer, M.; Graf, D.; Kiffe, M. Comparison of a Two-Dimensional Liquid Chromatography/Mass Spectrometry Approach with a ChipBased Nanoelectrospray Device for Structural Elucidation of Metabolites in a Human ADME Study Using a Quadrupole Time-of-Flight Mass Spectrometer. Rapid Commun. Mass Spectrom. 2007, 21, 937-944.

22. Pereira-Medrano, A. G.; Sterling A.; Snijder, A. P. L.; Reardon K. F.; Wright P. C. A Systematic Evaluation of Chip-Based Nanoelectrospray Parameters for Rapid Identification of Proteins from a Complex Mixture. J. Am. Soc. Mass Spectrom. 2007, 18, 1714-1725.

23. Ejsing, C. S.; Duchoslav, E.; Sampaio, J.; Simons, K.; Bonner, R.; Thiele, C.; Ekroos, K.; Shevchenko, A. Automated Identification and Quantification of Glycerophospholipid Molecular Species by Multiple Precursor Ion Scanning. Anal. Chem. 2006, 78, 6202-6214.

24. Qian, K.; Edwards, K. E.; Dechert, G. J.; Jaffe, S. B.; Green, L. A.; Olmstead, W. N. Measurement of Total Acid Number (TAN) and Tan Boiling Point Distribution in Petroleum Products by Electrospray Ionization Mass Spectrometry. Anal. Chem. 2008, 80, 849-855.

25. Håkansson, K.; Chalmers, M. J.; Quinn, J. P.; McFarland, M. A Hendrickson, C. L.; Marshall, A. G. Combined Electron Capture and Infrared Multiphoton Dissociation for Multistage MS/MS in a Fourier Transform Ion Cyclotron Resonance Mass Spectrometer. Anal. Chem. 2003, 75, 3256-3262.

26. Senko, M. W.; Hendrickson, C. L.; Emmett, M. R.; Shi, S.D.-H.; Marshall, A. G. External Accumulation of Ions for Enhanced Electrospray Ionization Fourier Transform Ion Cyclotron Resonance Mass Spectrometry. J. Am. Soc. Mass Spectrom. 1997, 8, 970-976.

27. Ledford, E. B. Jr.; Rempel, D. L.; Gross, M. L. Space Charge Effects in Fourier Transform Mass Spectrometry Mass Calibration. Anal. Chem. 1984, 56, 2744-2748.

28. Shi, S. D.-H.; Drader J. J.; Freitas M. A.; Hendrickson C. L.; Marshall A. G. Comparison and Interconversion of the Two Most Common Frequencyto-Mass Calibration Functions for Fourier Transform Ion Cyclotron Resonance Mass Spectrometry. Int. J. Mass Spectrom. 2000, 195/196, 591-598.

29. Blakney G. T.; Lam T. T.; Hendrickson C. L.; Marshall A. G. FT-ICR MS Data Station for Automated High Speed Data-Dependent Acquisition. Proceedings of the 52nd ASMS Conference on Mass Spectrometry and Allied Topics; Nashville, TN, May 2004. 